Article

\title{
Sustainable Wearables: Wearable Technology for Enhancing the Quality of Human Life
}

\author{
Jaewoon Lee ${ }^{1}$, Dongho Kim ${ }^{1}$, Han-Young Ryoo ${ }^{2}$ and Byeong-Seok Shin ${ }^{3, *}$ \\ 1 Department of Digital Media, Graduate School, Soongsil University, 369 Sangdo-ro, Dongjak-gu, \\ Seoul 06978, Korea; jwlee@gsclab.kr (J.L.); cg@su.ac.kr (D.K.) \\ 2 Division of Digital Media, Graduate School, Ewha Woman's University, 52 Ewhayeodae-gil, Seodaemun-gu, \\ Seoul 03760, Korea; hyryoo@ewha.ac.kr \\ 3 Department of Computer Science and Information Engineering, Inha University, 100 Inha-ro, Nam-gu, \\ Incheon 22212, Korea \\ * Correspondence: bsshin@inha.ac.kr; Tel.: +82-32-860-7452
}

Academic Editors: James Park and Han-Chieh Chao

Received: 21 February 2016; Accepted: 6 May 2016; Published: 11 May 2016

\begin{abstract}
This paper aims to elicit insights about sustainable wearables by investigating recent advancements in wearable technology and their applications. Wearable technology has advanced considerably from a technical perspective, but it has stagnated due to barriers without penetrating wider society despite early positive expectations. This situation is the motivation behind the focus on studies by many research groups in recent years into wearable applications that can provide the best value from a human-oriented perspective. The expectation is that a new means to resolve the issue can be found from a viewpoint of sustainability; this is the main point of this paper. This paper first focuses on the trend of wearable technology like bodily status monitoring, multi-wearable device control, and smart networking between wearable sensors. Second, the development intention of such technology is investigated. Finally, this paper discusses about the applications of current wearable technology from the sustainable perspective, rather than detailed description of the component technologies employed in wearables. In this paper, the definition of sustainable wearables is discussed in the context of improving the quality of individual life, social impact, and social public interest; those wearable applications include the areas of wellness, healthcare, assistance for the visually impaired, disaster relief, and public safety. In the future, wearables will not be simple data trackers or fun accessories but will gain extended objectives and meanings that play a valuable role for individuals and societies. Successful and sustainable wearables will lead to positive changes for both individuals and societies overall.
\end{abstract}

Keywords: sustainable wearables; wearable technology; the quality of life; public interest

\section{Introduction}

In recent decades, wearable technology has ignited a new type of human-computer interaction with the rapid development of information and communication technology. Wearable technology assigns the attributes of mobility and connectivity to users so that users can access online information conveniently and communicate with others (or other things) immediately while moving [1]. The technology is characterized by "wearable", as its name implies in contrast with the concepts of simply holding portable compact computing devices. Wearable computers are inherently different from portable computers such as sufficiently small laptops or separated desktop computers; accordingly, it is highly important to understand the value that can be acquired from using wearable technology by users. An early experimental study conducted by Steve Mann, one of the pioneers in wearable technology, started by strolling around the street carrying a computer and wireless equipment that 
looked cumbersome and heavy [2-4]. In contrast with current wearable devices, which are light, unnoticeable, and beautiful, early wearable devices looked entirely different, but the wearable concept was clearly exhibited by "wearing" the devices on the user's body to extend the user's mind and body practically [3].

Today, wearable technology has faced a critical challenge. In contrast with the positive prospects of wearables from various sectors in its early days when wearable technology started being commercialized, many survey results show that its proliferation rate into public society has not met expectations [5-8]. The results found that one-third of American consumers who have owned a wearable product stopped using it within six months. Moreover, half of them no longer use it while one in 10 American adults own some form of wearable devices. What is the problem with wearables? To expand its use with the public, it is necessary to pay attention to the essential principle of wearables - that they should be closer to users' daily lives than any other devices. Nonetheless, the meaning of the term "wearable" tends to be mentioned as only the restrictive definition, which is fitness tracking devices that are accessorily used to maintain individual health status worn on a wrist. Major wearable devices such as smartwatches and fitness bands that have launched in the market up until today include the features of activity tracking and the functional sensing of biometric information such as the heart rate and body temperature. Services provided by wearable devices can display not only the current heart rate and activity but also how many times the user turned over during sleeping. However, apart from whether these functions and services are universal requirements from a user's perspective, it is not yet clear whether these functions and services are appropriate applications for the effectiveness of wearable devices. For example, although collected information is displayed in some applications that work with smartphones, merely listing the measured data is not sufficient to maintain the use of wearable devices. The issue of the effectiveness of wearables is one of the claims raised from the early days of noticeable product launches in the market up until now. Donald Norman, a cognitive scientist and pioneer in applying human cognition to design, mentioned that "much of what is being done with wearable devices is happening simply because it can be done [9]". What Norman pointed out is that the current wearable technology trend mainly focuses on core technology development while ignoring practical extensions of mind and body through technology, which is an essential inherent objective of wearable technology. Nonetheless, it is now quite promising that more researchers and companies are focusing on researching wearable applications and services that can provide the best value from the users' perspective.

In this paper, human-oriented wearables that focus on the value of sustainability, sustainable wearables, will be discussed, with particular attention on recent case studies into wearable technologies and services that seek to improve the quality of life, which include social impact and public interest (Figure 1). Sustainable wearables defined in this paper refer to the wearable technology and its applications with a value of sustainability having a positive impact on the improvement of quality of life, social impact and the public interest. Although a large number of studies have been published about various wearables, this paper will focus on innovative and experimental case studies rather than existing commercial technologies that exhibit limited applications of said wearables. This is because we are aiming to discuss how continuously evolving wearables influence positively on human life and environment through the keyword of sustainability. 


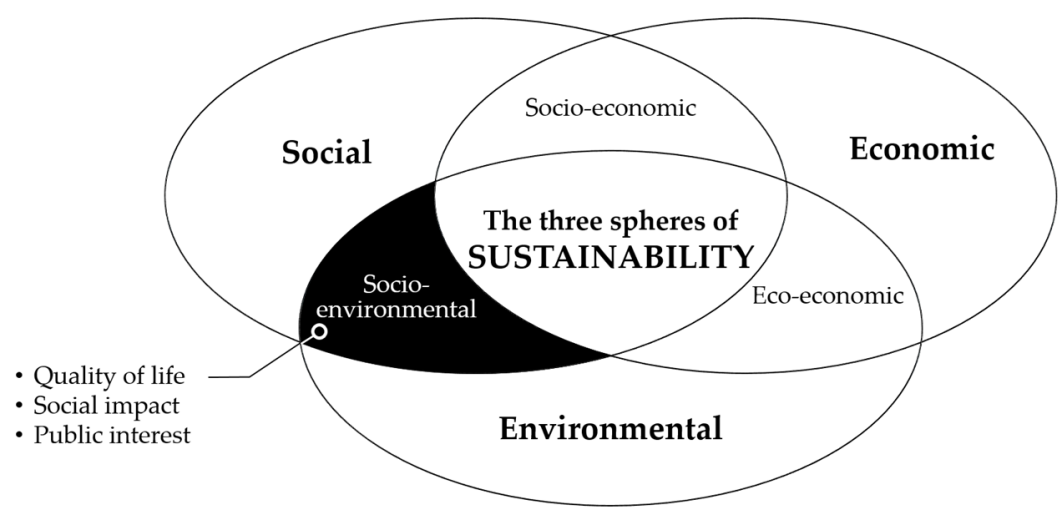

Figure 1. The value of sustainability for wearables covered in this paper.

\section{Technological Trends in the Field of Wearables}

Monitoring and measuring physical activities and physiological statistics, sensing environmental status, real-time data collection, alarming and communication, and manipulating and controlling external systems are some of the wearable applications included thus far. Furthermore, many innovations will be investigated to accommodate all issues about users' requirements not only for professional jobs such as medical doctors or firefighters but also for the vulnerable such as disabled persons and the elderly for future wearables. Not only the interactions between humans and computers and between humans and machines but also all the demands from existing data analysis, context-aware technology, wireless sensor networks and intelligence ambient applications to adaptable user interfaces are at the center of innovations. In this regard, this chapter briefly describes the main technological trends in the current wearable technology fields (Figure 2). Wearables can have various technical areas, but four primary technical keywords of wearables are selected in this paper. The keywords are based on the report by LexInnova Technologies, the patent analysis company. After analyzing applications and the trend of registered patents using its exclusive patent analysis tool, LexScore ${ }^{\mathrm{TM}}$, LexInnova has classified the major technology areas of wearables on the basis of the data [10]. From the results of this report, we have selected human data tracking, human big data analyzing, Internet of Things, middleware for wearables as the main keywords for wearable technology development.

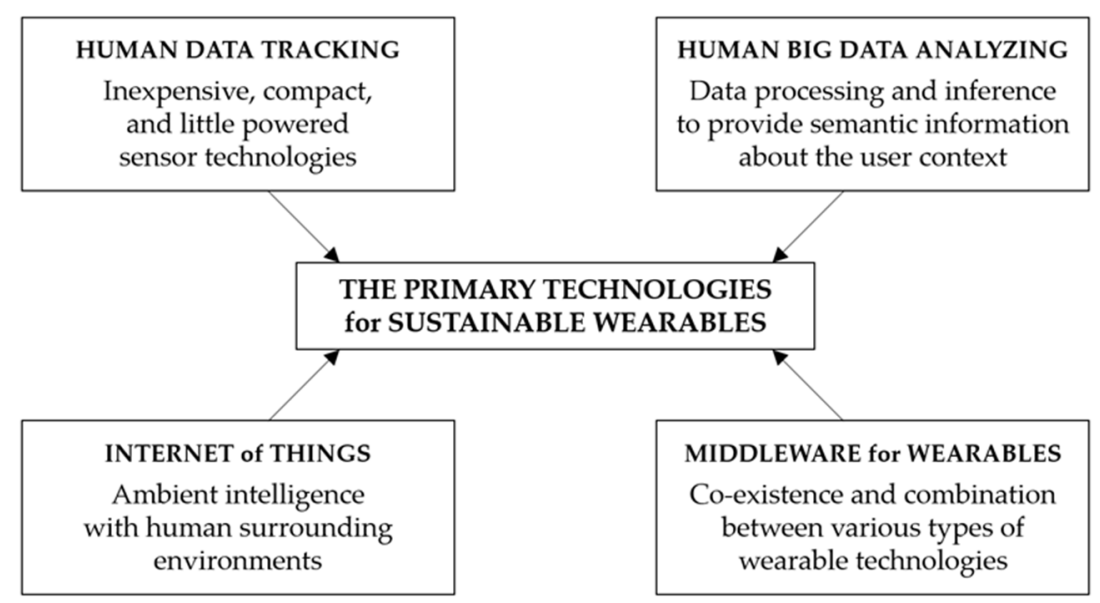

Figure 2. Primary technological keywords for the development of sustainable wearables.

\subsection{Quantified Self as Human Data Tracking}

Wearable devices and sensors have been developed to track physical body information. A variety of wearable devices have been launched in the market to achieve various purposes with the 
development of sensing technologies. One typical example is an application that constantly measures movement distance and movement conditions of users over time through motion sensors included in wrist-wearable devices, and displays the measured results [11]. Moreover, measuring the intake and consumption of calories, tracking sleep, postural correction, blood pressure, and heart rate are the most fundamental applications of the current wearables field. As such, wearable applications started by quantifying various human activities (consciously or unconsciously) numerically in daily life. Over the past few years, more wearable devices have been introduced according to their purpose with increasing performance. As a result, the demand for them to quantify individual daily lives by themselves has increased [12]. Along with this demand, more studies of the methods to improve the quality of life by analyzing individual conditions have been conducted for application in real life, which is called the quantified self. Targets whose movements are tracked include various types of personal information such as physical activities performed and environmental information [13].

Various types of sensors have been mounted on commercially available wearable devices. Wearable sensors are used to collect data about physical activities and physiological status for monitoring user conditions. Basically, inertial sensors including three-axis accelerometers and gyroscopes are included in determining user movement sensing and tracking. Often, magnetometers are used along with inertial sensors to improve movement tracking. Wearable sensors perform user tracking, monitoring, and diagnosis through motion sensing and both physiological and biochemical sensing [14,15]. Cardiotachometers that measure heart rates [16], bio-impedance sensors that measure blood flow resistance and skin tissue [17], and electromyography sensors that measure muscle status changes through hand movements [18] can also be employed. In addition to the above measurements, respiratory volume, skin temperature, skin conductance, and blood volume pulse can be measured through wearable devices [19]. From a design viewpoint, measurements of bodies' physiological and biochemical signals can be more complicated than physical motion tracking, since they require data collection, processing, and analysis, mainly in relation to body fluids. In this regard, much attention has been paid to sensors for physiological tracking among wearable technology studies in recent years [20]. In the past, continuous monitoring and the measurement of such physical and physiological parameters were only possible at hospitals or research laboratories with specific settings. However, the advancement and proliferation of wearable technology have made such measurement possible anywhere [21].

Modern wearable devices are inexpensive, compact, and require little power consumption; these advantages are quite attractive to their users. The technological advancement of wearable devices enables measurement and tracking for physical activities and physiological status of human in any place on the body. It can be worn on any part of the body such as the head, ear, eye, arm, trunk, finger, neck, and foot to perform tracking tasks [22]. It can not only be attached to external surfaces or in proximity to the human body but also even inside the human body. Some devices are even equipped with sensors to trace activities that can be located inside the body as they pass through the intestines $[23,24]$ in addition to functioning as an artificial ear and cardiac regulator that can be transplanted into the body. In the past, the size of front-end devices and sensor hardware that collect physiological and physical data appropriate for long-term monitoring was one of the main obstacles in the adoption of the sensing technology used in wearable devices. However, such difficulty has been overcome as the size of the electronic circuitry and sensors included in wearable devices have become miniaturized. Advancements in the microelectromechanical system field have not only enabled various types of sensors to collect physical and physiological data but also the implementation of very tiny wearable devices that can include units through which data can be transmitted remotely. Ultra-tiny flexible circuit-applied wearable devices are an example of such technology. Moreover, much improvement has been made on minimizing the fatigue and skin trouble felt by users due to long-term wearing or attachment, and this trend has introduced wearable sensor-embedded smart clothes [25-27]. 


\subsection{From Data to Information and towards Context-Awareness}

Individualized big data has become a reality with the introduction of wearable computers. The exponential increase in wearable devices embedded with various sensors incurs different types of personal data production [28]. These data are highly unique due to the personalized characteristics of wearable devices and have the high potential that can obtain insights about a user's conditions. However, since they generate a vast amount of big data that cannot be handled with the current technology, often most of the data may not be sufficiently utilized. Wearable devices, including wrist type, headsets, glasses, and smartphones and devices such as medical equipment, collect data constantly in real time through user input and sensors. For example, a heart rate monitor can collect 250 samples per second and produce a number of gigabytes per person per month [12]. Thus, data should be processed and inferred to provide normal semantic information about the user context. In relation to this, much attention has been paid to processing the vast amount of data collected via physical and physiological tracking on individuals via wearables and analysis of correlations as new core technology trends in wearable computing $[7,29]$. Methods of extracting and providing related information that can be easily accessed and utilized by users from the data flood collected via wearable systems that monitor user conditions have also been recognized as an important component of the technology. Data should not be collected simply for the purpose of collection. In other words, data collection should not be the sole aim; instead, data should be used as a medium that facilitates positive changes in individuals' lives. Flexible and dynamic data can be a source of power that can provide useful information and context for users more easily than ever before. Dave Evans, Cisco's chief futurist, said that future wearables should evolve into "aware-able" that can be context-aware rather than just play a simple tracking role [30]. This means that wearables should ultimately provide meaningful and appropriate services to users by identifying situations and contexts with high quality and interpreting user data into the information level. Wearables-based user context-aware technology can be achieved through adaptable data analysis technologies such as signal processing, pattern recognition, data mining, machine learning, and other artificial intelligence-based methodologies [31,32].

Wearable applications need to detect appropriate and required services according to user context depending on specific constraints and conditions. Norman mentioned in his article, the Paradox of Wearable Technologies, that "when technologies are used to supplement our activities, [and] when the additional information being provided is of direct relevance, our attention can become more highly focused and our understanding and retention enhanced. When the additional information is off target, no matter how enticing it is, that's the distracting and disruptive side [9]." This means that when wearables provide unclean and indiscriminate information by ignoring user context, they can have an adverse effect on people. The inappropriate application of technologies despite their assistance in daily life can increase fatigue when accepting information and degrade work productivity. Although the following statement may be somewhat exaggerated, recent wearable applications do not edit information contents before displaying it to their users. That is, it seems that the content provided by wearable devices is just a bunch of data sets, with the expectation that users will find them useful or interesting. With the only visually appealing graphs and charts, data are displayed to users. This is a typical and repetitive mistake that has been made by many wearable device companies $[7,8]$. In the midst of rapid growth in the wearable market, many suppliers claim that their wearable devices and applications are providing more functions and performance than others while making marketing efforts to be noticeable to potential users. However, little effort has been done to make wearables more useful from the user's standpoint. However, users should be aware to some extent that they need "more compact information" or "at least less useless information" in some cases. The ultimate purpose of wearables is to provide necessary information to users when they need it. Today's wearable sensors are all excellent in collecting data, but the ability to analyze and utilize data will be a deciding factor in the success of future wearables. 


\subsection{Ambient Intelligence, Internet of Things}

Wearables provide assistance to users via devices independently or through simple smart accessories. However, the recent trend of wearable devices is integration with surrounding environments, although wearable devices still perform their jobs as independent entities [33]. Information collected through wearable sensors is augmented with information gathered from environmental sensors. The results of data collection using wearable devices can be strengthened by other sensors installed to determine the type and degree of activities performed by individuals in a particular environment. For example, innovative solutions to detect emergencies at home can be achieved by the integration of the monitoring of residents' critical physical parameters that can be collected directly from wearable devices with the tracking by other devices installed in the house or electronic appliances. Furthermore, remotely monitoring causes of potential risk factors around environments and spaces where people are located rather than focusing on individual data can make their lives easier and more comfortable. If necessary, operating heaters, coffee machines, or gas controllers remotely can be basic applications as in some examples [34]. Sensors embedded in electronic devices, exit doors, or windows at home can be integrated into home control systems that are provided for personal safety and security solutions [11]. In these ways, ambient intelligence under ordinary daily scenarios can be used to collect data from various sensors to understand the users' environments. All data are employed as they are combined and processed to enable users who operate easy-to-understand interfaces to make decisions.

Much attention has been paid to the advancement of wearable computing technology that can quickly change our society into a hyper-connected society. That is, with the popularity of wearables, the future world will extend its connection range from the Internet of People (IoP) in the past to the Internet of Things (IoT) and from there to the Internet of Everything. In the future, the era of living in the intelligent environment, where not only people, things and spaces, but also virtual worlds are integrated will come. In the current technological development that is directed towards creating a hyper-connected society, wearable devices will play a role as a tool that connects people with the world. The connection of wearables with other external environments induces potential possibilities that bring various types of prototypes and their deliverables. This can be understood more extensively from the viewpoint of IoT or ubiquitous computing. The role of wearables is not limited to just trackers of physical body status anymore; it can be extended to search for utilization methods by linking with external services after collecting various data about personal information generated by other apps and devices [35,36]. In this process, wearable technology will have a significant role in achieving objective value. The ultimate goal of wearables, often proposed as the future direction, is a natural connection to other devices. Once the IoT becomes popular, the roles of wearables can exponentially expand in their environments. The added values that will be created by communication between surrounding things and machines that can identify user status the best will be numerous, because wearable devices are an essential element in recognizing user contexts to construct human-oriented environments in the IoT. Ultimately, users will have more potential when they are connected to various external services over the IoT environment.

\subsection{The Requirement of Middleware Technology}

One of the problems with implementing services that support users through wearables is the consideration of appropriate co-existence between various types of heterogeneous systems. In the future, it will be natural to combine a large number of wearable devices. It is necessary to synchronize data without problems with all types of human tracking data over heterogeneous environments including various devices, operating systems, and applications for both wearables and ambient intelligence to provide appropriate and useful user experience based on user context. To do this, it is desirable to abstract hardware functions and protocols from the higher layers with regard to various types of wearable devices or platforms. As a result, it is also highly important to study the middleware that controls and manages by integrating a large number of sensors. Middleware is 
defined as an abstract layer between sensors and the application layer. Furthermore, it can refer to software and tools that can resolve the complexity and heterogeneity of base hardware and network platforms while facilitating the management of system resources and increasing predictability of application execution [37,38]. It would be highly useful to process both user parameters collected through various sensors and all the measured environmental data at the middleware level. Although the requirements of wearable applications can differ from application to application, they generally include high flexibility, reusability, reliability, localized extensibility, adaptability, and contextual factors [39]. The adoption of middleware for wearable systems enables more efficient development of wearable applications that combine types of new devices without various external interferences.

The main element that provides wearable services in an individualized mode is the possibility of collecting multiple data from not only body-worn devices but also from environments where users are located. Most wearable applications are limited to body-worn sensors, but they will use multi-sensors from sensor networks where body-worn sensors and environment sensors are integrated. In wearables, a sensor network system is a crucial element for communication between user and environment and also between environments [40,41]. These devices transmit data to wireless sensor network nodes equipped with communication interfaces, which do not need complex cable connections. Instead, a bigger problem is the lack of the computer resources required to process personalized data, including environmental data, at the middleware level for wearable systems. A wearable sensor collects a large amount of raw data about physical behavior and the physical states of users. The need for big data analysis solutions that extract hidden tendencies and conditions from the collected data has arisen. It is very difficult to manage, store, and process raw data in real time or near-real time; the high bandwidth and storage requirements incurred by real-time collection can create a serious problem [42]. Nonetheless, requiring that users carry high-performance computing devices at all times to process data would not be a preferable solution. Although compact wearable devices with central processing units (CPU) such as smartphones or smart watches have advanced significantly, their performance is not yet sufficient to analyze big data that is continuously being collected. To solve this problem, an approach of accepting the cloud paradigm has been highlighted in recent years [43]. In this approach, main human data is analyzed at an internet-based remote system; through this, the insufficient capabilities of hardware, whose resources are limited, which can occur due to energy depletion, processing power, or memory size constraint, can be overcome. The need for real-time multitasking and the limitations of both computing performance and data storage capacity require data storage and the use from/to cloud-based remote servers. As wearables become increasingly cloud-based systems, wearables can better achieve low power consumption, platform-independent rapid deployment, and universal connection. A distributed process system over the cloud of such advantages has arisen as the primary technology trend to improve the limitation of middleware for wearables.

\section{The Approach from Wearables for Sustainability to Enhance the Quality of Human Life}

Recently, users and developers have started to realize that future wearable technology should evolve from its current state of only collecting simple physical activities or biometric data. Furthermore, prospects claimed by several researchers that wearable computers would not only improve the quality of life but influence all aspects of life have been realized today. The current wearable domain has attempted to improve the quality of life from sustainability viewpoints and adopt human-centered wearable applications and services for social public interest and safety beyond the concept of measuring physical body or environmental states while tracking and monitoring outcome data about various events which are changing over time, as mentioned previously. This approach may be explained by the model of Figure 3, which is based on the social ecological model [44,45]. In this regard, this chapter will discuss the concept of wearables in terms of sustainability. 


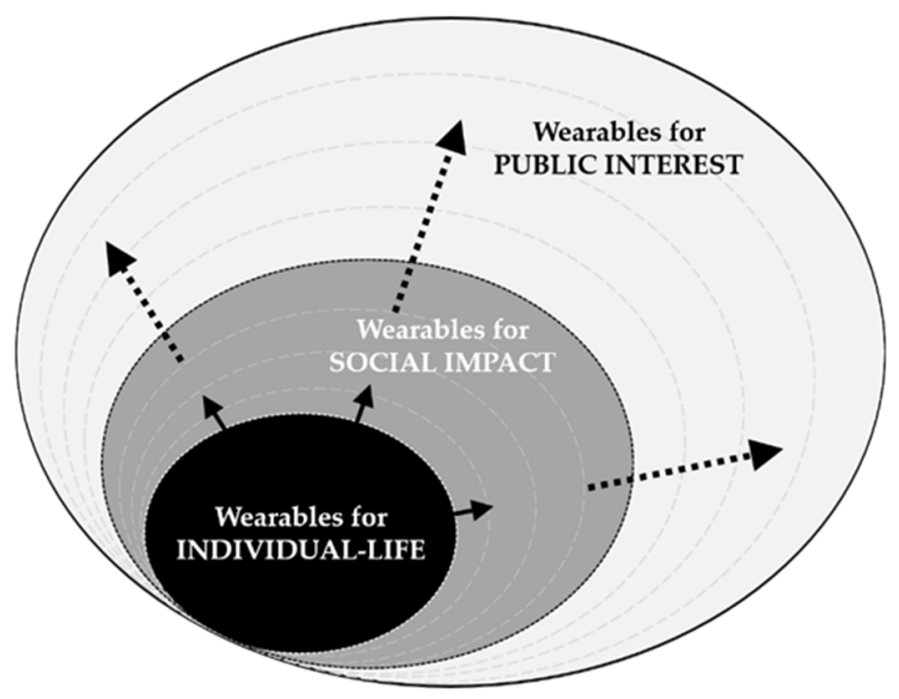

Figure 3. The concept of sustainable wearables to enhance the quality of human life.

\subsection{User Centric Wearable to Enhance Quality of Individual Life}

The initial adoption of wearable technology, which is aimed at improving individual quality of life, raised expectations of imaginable utilization such as not only improvements in personal work efficiency and health management but also future-oriented scenarios by many people in this field. However, it still seems that there is a long way to go to achieve the functionality that optimistic imaginations envisioned in the early days. Many wearable users have reportedly given up using their devices within six months after purchasing wearables that are categorized as commercial fitness [6]. The reasons that cause this problem include the technological issues mentioned earlier and various user experience factors related to the lack of understanding of user requirements, wearing sensation, and aesthetics in the cultural context $[7,8,46]$. Ultimately, the wearables preferred by users are light and comfortable as if they are not being worn, and provide only appropriate information whenever needed like a personal assistant. It is true that various areas of component technology such as the development of human body - friendly materials, the enhancement of sensor technologies, big data analysis, and artificial intelligence technologies should be supported to give a complete user experience. Although studies on these technologies have been conducted for a considerably long time, it may take a long time to commercialize technologies so that users feel satisfied. It is necessary to consider how to improve the usability and effectiveness of wearables using methods from the limited current technological level and the inherent aspects of wearables for how to satisfy users while also performing in-depth studies from the user perspective. Thus, it is important to base the design on users' experiences. This will not only provide new possibilities and interaction methods in the wearable environment but also enable wearable-based user-aid services, which will be more adaptable and natural to improve users' quality of life. Next, the items that need to be considered in user experience-based design of wearables are discussed to improve the quality of life [47].

- Unmonopolizing: Interaction with the wearable devices should be a secondary activity rather than a primary focus of attention.

- Unrestrictive: Wearable devices should be designed for daily mobility activities such as walking or jogging.

- Observable: Wearable devices should respond immediately to other media use such as display, sound, and motion.

- Attentive: Sensors that can be aware of the surrounding environment should be wearable.

- Communicative: Wearable devices should allow users to connect and communicate with other users, surrounding things, and webs. 


\subsection{Wearables for Social Impact and Public Interest}

By adopting wearables, efforts that focus on both quality of human life improvements and sustainability values based on social impact can be created. Today, many people tend to regard social impacts as purely charitable work through financial or human resource support. However, technologies and innovation can also be understood as a critical approach that can play a decisive role in growing the social impact sector. The United International Children's Emergency Fund (UNICEF) proposed an interesting contest about wearable design called the "Wearable for Good Challenge" and awarded prizes in the autumn of 2015. The topic of this contest was to design solutions for innovative and inexpensive wearable devices and sensor technology that can be helpful for people living in resource-limited environments (such as children and women in developing countries) [48]. In addition, it was to facilitate wearable solution development for social good by collecting communities of social impacts, designs, and technologies as one. In light of these objectives, UNICEF seems to interpret the definition of wearable technology in a broader sense. UNICEF claimed that the current roles of most wearables that focused on helping users by improving productivity were short-sighted applications and only a fraction of the work that could ultimately be achieved by wearables [49]. UNICEF have adopted and applied innovative wearable technologies at its social impact activities. The claims and activities by UNICEF make us think about the goal and ethical implications of wearable research. This means that wearables are not only helping users to become more productive but are also improving the quality of life, thereby evolving into a role of promoting social public interest. In this regard, UNICEF proposed the following prior conditions for future wearables [48].

- Cost Effective: Can be executed en masse in low-cost areas, with a demonstrated business case and need

- Low Power: Runs off a battery, has a long battery life (possibly alternate energy, is power efficient where there is no power)

- Rugged and Durable: Is waterproof, shockproof, weatherproof, heat resistant, easily stored, and built to last

- Scalable: Can be applied to various environments and communities, consider larger ecosystems, easily produced/developed, easy to use, and easy to maintain (fixed/addressed by local skill)

Although wearables are currently perceived as interesting tiny portable devices, we believe they will get the opportunity to spread all over the world for critical fields such as responding to social crises in the near future. Wearable technology will likely play a major role in helping public protection activities, which is highly important both nationally and socially. These proposals should not be limited to the socially vulnerable; they need to be considered as broad as possible regarding public protection in order to ensure individual safety and comfort and to maintain social stability. In this context, the current price of wearable devices makes it difficult for them to spread throughout society. It would be too expensive for organizations to replace damaged devices or parts when they are broken during use. This problem is a critical factor and may even cause organizations to abandon the use of wearables despite their potential advantages. Ensuring power and durability is also a highly sought requirement in terms of the safety of disaster relief personnel and law enforcement officers entering dangerous situations that require intensive concentration. If batteries embedded in devices are large or heavy enough to restrict relief activities or if wearable equipment is easily damaged due to tough environment conditions, their lives are put in jeopardy. Thus, ensuring power and durability is an essential requirement that wearables should provide. Another consideration is that easy-to-use interfaces should help responses to the urgent situation quickly and the issue of unreachable or out of range network infrastructure should also be studied critically.

These approaches will be excellent guidelines for wearables to enter a new domain for the social impact on the socially vulnerable, public protection, and improvements in the quality of individual life, which has not yet matured completely. Wearables that aim to achieve a broad range of objectives from user experience improvements and individual quality of life to social public interest should not focus 
on the myopic perspective that concentrates on technology development affecting only a single area. Instead, it is necessary to have a comprehensive approach that takes overall ecosystems surrounding individuals (or users) into account. Successful sustainable wearables will lead to positive changes in systems for both individuals and society overall.

\section{Applications of Sustainable Wearables}

The first ten years of wearable technology emphasized research on the engineering of wearable sensors and systems. However, future wearables are focused on wearable technology applications that are directed to improvements in the quality of life and the value of life. In this chapter, specific wearable applications are discussed in terms of sustainability focusing on improvements in the quality of life and social public interest. The following applications, as it deems appropriate for the concept of sustainable wearables mentioned in Chapter 3, were selected through an extensive literature survey by Google Scholar and others.

\subsection{Wellness and Healthcare}

The fitness field is one of the most familiar wearable applications that we experience every day. In practice, the majority of related products including software and applications have been launched in this field. Furthermore, there have been many attempts to converge wearable technology for expanding this market [22]. By combining position-based tracking such as a global positioning system (GPS), motion tracking using accelerometers and gyroscopes, and physiological tracking via heart rate monitors, which are the most fundamental utilization included in wearable devices, user logs such as distance moved, calories consumed, and average speed can be calculated. Furthermore, the use of physical posture detection sensors can propose the optimum exercise routine after examining whether users are exercising correctly [50]. Wearable technology encourages active and healthy lifestyles, so it can be utilized to monitor physical activities to improve the health and physical strength of those who need exercises such as obese people or those who lack exercise due to work; it can also facilitate clinical intervention [51].

Many research groups have looked into the possibility of wearables to accelerate healthcare beyond simple fitness assistive applications used by individuals. An extensive study on remote monitoring applications based on wearables has been conducted in the healthcare field, mainly for the elderly who have difficulty in their daily lives and patients with movement disorders due to fatal chronic diseases such as Parkinson's disease, stroke, or dementia [14,42,52]. These patients need constant assistance from guardians such as family members or caregivers because they cannot live normally without help. Hence, related studies aim to improve their quality of life by monitoring their health condition, behavior, and signs of worsening symptoms, while also providing information about condition changes over time, thereby enabling independent and comfortable daily life activities. In addition, it helps with the management of chronic diseases by exchanging information about patients' health conditions in real time among patients, doctors, and family members. Wearable devices attached to patients play an important role in improving diagnosis and treatment methods by monitoring physiological data such as heart rate, blood pressure, oxygen saturation, respiration rate, body temperature, and electrical skin reaction over a long time [50]. The parameters extracted from these measurements can provide indices for health conditions of patients with significant diagnostic value [50]. Until comparatively lately, the constant monitoring of physiological parameters was only possible at hospitals, but patient data now can be monitored in real time during normal home life. The technological development can help doctors determine the symptoms of patients more accurately.

The wearables can now be utilized even in the remote rehabilitation treatment field. For example, intensive long-term rehabilitation is a major factor in ensuring the recovery of reduced exercise function after the onset of a stroke. Tracking the change in conditions of exercise functions can be utilized as a feedback means to guide appropriate rehabilitation processes [53-55]. Furthermore, remote home-rehabilitation applications based on wearables increase the time that patients can stay 
at home instead of visiting hospitals for the purpose of treatment, thereby reducing treatment costs. These remote applications can also be utilized to monitor the safety status of patients with movement disorders. A broad range of wearable devices that are integrated with wireless networks can track and analyze patients' movement status such as walking, spotting movement difficulties, thereby automatically sending alarms or urgent call messages to their family members or caregivers when patients fall or experience a sudden shock [56-58]. These applications can both detect physiological states of patients and enhance their sensitivity of detection by combining with motion sensors such as accelerometer mounted in wearable sensors in the patient's home environment. They may help patients with their daily living and reduce the stress on family members by providing accurate information after analyzing data streams in order to monitor health conditions, daily living activities, and urgent situations.

\subsection{Aid for the Disabled}

A high percentage of visually impaired people are always exposed to the risk of falls and collisions in the street [59]. Wearables can give visually impaired people the opportunity to extend their daily living areas safely and easily, and this can also help other vulnerable people whose daily lives are limited to their homes. These applications will play a role in providing useful information that can replace or overcome deficient visual senses [60,61]. For example, wearable devices in which Wi-Fi, Bluetooth, GPS, and magnetometers are embedded along with various other sensors to collect environment information can help visually impaired people navigate streets with confidence despite their weak vision. Through a combination of wireless sensor network technology and ambient intelligence technology, various pieces of information about the surrounding environment are transmitted to a wearable device, allowing them to detect the presence of obstacles, thereby giving notice of risks in advance while the visually impaired person is walking. It is also now possible to have an Internet connection and wireless communication via 3G or LTE (Long Term Evoltution) that covers a wide range of areas, so that constantly updatable location-based information such as the current location and direction, surrounding buildings, and travel path to a destination can be provided to a user through built-in speakers in the wearable. It is also possible to re-route paths to a destination if a visually impaired person goes in the wrong direction or gets lost on the way and gives notice to family members if the visually impaired person is hit by a car or another such emergency situation occurs. There are even cases in which visually impaired people have participated in active sports such as marathons through the assistance of similar wearable devices [62]. Images collected from high-performance camera sensors embedded in glass-type wearable devices enable more accurate recognition of objects in front of a person through computer vision technology [38]. This type of application helps visually impaired people operate electric appliances such as microwaves or washing machines as well as perform some detailed operations accurately such as pushing buttons on an automated teller machine (which might be easy for normal sighted people). The development of various types of wearable applications for visually impaired people can help them do many important tasks in their daily lives, thereby making their lives more complete.

\subsection{Disaster Relief and Public Protection}

Disasters have been on the rise around the world, affecting economies, infrastructures, and creating serious human casualty statistics. Despite the rapid development of technology and science, humans are still vulnerable in disasters. Responding to disasters quickly is one of the most important issues both nationally and socially. In this regard, there are studies on the use of wearable technology during disasters. Several studies on wearable applications that help response to urgent situations of people who work in dangerous and hostile environments appropriately and monitor their safety status have been published [63-65]. The typical accomplishment of the related studies is the development of wearable devices and clothing for emergency disaster relief personnel such as firefighters through wearable sensor networks and body-area network technologies used to monitor 
individual physiological status and the statuses of surrounding environments. These wearables are miniaturized with low power consumption, and they include sensors and data transmission devices that can endure the harsh conditions. Through these wearables, parameters related to the biological signals and health status of firefighters in addition to environmental variables such as outdoor temperature at the disaster area, presence of toxic gases, and heat flow applied to the worn clothing can be constantly detected. These data are processed and analyzed remotely in real time, thereby facilitating monitoring fatigue level and the focus state of attention felt by relief personnel under critical situations where a high degree of concentration is required. Relief personnel can also receive appropriate treatment before their critical biological signals reach fatal conditions and assistance can respond rapidly and accurately to changes in relief field circumstances.

In another example, people who maintain order such as police officers can get some help [66]. Police officers who conduct early response duties such as law enforcement have employed light wearable devices that are attached to heads, wrists, hands, pockets, shoulders, or weapons so that their hands remain free without interrupting their tasks. In the future, more wearable devices will be employed as daily routine devices for patrol officers. On-body evidence capture is one of the most well-known wearable technologies used for law enforcement; with the development of wearable technology, on-body cameras have started integrating with other various functions provided by wearable devices in general. Built-in liquid crystal head-up display, wide-view camera, microphone, touch pad, and smart network devices for data transmission, which are developed for hands-free technology for police officers were developed as a glass shape device (similar to Google Glass) for on-body evidence capture. These devices can be used for relatively simple tasks such as vehicle plate recognition and driver license examination as well as criminal identification through facial recognition technology by processing and analyzing data constantly collected using video and audio gathered automatically from a remote place rapidly. Furthermore, the measurement of physiological status can be applied to police uniforms or bulletproof vests for police officers on duty in dangerous situations such as car chases or shootings. Critical information such as heart attacks or other various fatal incidents that occur during car collisions or shootings can be transmitted to other colleague police officers or police departments, helping them respond to urgent circumstances quickly. If a policeman is shot by a suspect while chasing them after getting out of their car, wearables that are capable of wireless network communication with various sensors may increase the probability of finding the police officer's location quickly, thus saving their life.

\section{Conclusions}

This paper provides a comprehensive review of wearable technology fields in terms of sustainability, focusing on improving the quality of life, social value, and public interest. This paper first selected the following subjects as the main wearable technology trends in recent years: Sensor devices that track and collect human data for user monitoring, context-aware technology that processes and analyzes collected data and provides useful information to users, the evolution into wearable technology that is extended through a combination of users' physical bodies and the surrounding environment, and middleware technology that provides efficient wearable applications. In addition, this paper discussed the requirements and future directions of sustainable wearable technology. Finally, wellness, healthcare, disaster relief and public protection as well as case studies on wearable applications for the disabilities were briefly summarized. Through the above discussions, the inherent meaning of wearables, which extend the mind and body of individuals to direct the sustainable wearable technology was examined, and positive effects and great potentials, which aimed to improve the quality of individual life and social public interest, were verified. This paper emphasized that wearables in the future will not be simple data trackers or interesting accessories but should have extended objectives and meanings that play valuable roles for both individuals and societies.

The future wearables will collect more accurate and diverse individual information than ever and will be utilized in wider areas. They will also provide accurate and needed information at the 
right time to the user. Future wearables will be easier to use, more convenient, wearing them will not be bothersome, and they will operate non-invasively and eventually be invisible. However, several obstacles must be overcome for wearables to achieve their potential completely. The technological and policy-related obstacles that must be overcome are as follows: the potential risk of private information leakage due to wearable devices' capabilities to collect a vast amount of information; strict and cautious regulations on wearable technology's participation in medical activities, whether directly or indirectly; the development of power supply devices that are tiny, stable, and have longer battery life; the expansion of wireless communication range; integration with non-standardized sensors and platforms; improving the reliability of sensors, and the improvement of artificial intelligence [67-71]. Despite the above challenges and obstacles, various studies on sustainable wearables will need to be carried out to solve the above issues. In Chapter 3, we mentioned that successful sustainable wearables will lead to positive changes in both individuals and in societies as a whole. From the viewpoint of sustainable wearables, both for the quality of life and for the social value and public interest, which are the theme of this paper, a new direction will be found to pioneer a new domain of wearables.

Acknowledgments: This research was supported by the Next-Generation Information Computing Development Program through the National Research Foundation of Korea (NRF) funded by the Ministry of Education, Science and Technology (No. 2012M3C4A7032182).

Author Contributions: Jaewoon Lee and Dongho Kim conceived and designed the main idea of this paper; all authors, Jaewoon Le, Dongho Kim, Han-Young Ryoo and Byeong-Seok Shin wrote this paper.

Conflicts of Interest: The authors declare no conflict of interest.

\section{References}

1. Billinghurst, M.; Starner, T. Wearable devices: New ways to manage information. Computer 1999, 32, 57-64. [CrossRef]

2. Mann, S. Wearable computing: A first step toward personal imaging. Computer 1997, 30, 25-32. [CrossRef]

3. Mann, S. "Smart clothing": Wearable multimedia computing and "personal imaging" to restore the technological balance between people and their environments. In Proceedings of the Fourth ACM International Conference on Multimedia, Boston, MA, USA, 18-22 November 1996; pp. 163-174.

4. Mann, S. Wearable, Tetherless Computer-Mediated Reality: Wearcam as a Wearable Face-Recognizer, and Other Applications for the Disabled; AAAI: Palo Alto, CA, USA, 1996; pp. 62-69.

5. Herz, J.C. Wired. Available online: http://www.wired.com/2014/11/where-fitness-trackers-fail/ (accessed on 13 January 2016).

6. Ledger, D. Inside Wearables_-Part 2; Endevour Partners: Cambridge, MA, USA, 2014; pp. 2-3. Available online: http:/ / endeavourpartners.net/assets/Endeavour-Partners-Inside-Wearables-Part-2-July-2014.pdf (accessed on 16 April 2016).

7. Clawson, J.; Pater, J.A.; Miller, A.D.; Mynatt, E.D.; Mamykina, L. No longer wearing: investigating the abandonment of personal health-tracking technologies on craigslist. In Proceedings of the 2015 ACM International Joint Conference on Pervasive and Ubiquitous Computing, Osaka, Japan, 7-11 September 2015; ACM: New York, NY, USA, 2015; pp. 647-658.

8. Lazar, A.; Koehler, C.; Tanenbaum, J.; Nguyen, D.H. Why we use and abandon smart devices. In Proceedings of the 2015 ACM International Joint Conference on Pervasive and Ubiquitous Computing, Osaka, Japan, 7-11 September 2015; ACM: New York, NY, USA, 2015; pp. 635-646.

9. Norman, D. MIT Technology Review. Available online: http://www.technologyreview.com/news/517346/ the-paradox-of-wearable-technologies / (accessed on 13 January 2016).

10. Wearable Technology: Patent Landscape Analysis; LexInnova Technologies: Cupertino, CA, USA, 2016; Available online: http://www.wipo.int/export/sites/www/patentscope/en/programs/patent_ landscapes/documents/patent_landscapes/lexinnova_wearable.pdf (accessed on 16 April 2016).

11. Agaskar, A.P.V.A.; Mithagari, A.; Mhatre, A.; Shetty, N. Internet of things: Home automation and surveillance system. Int. Educ. Sci. Res. J. 2016, 2. Article 4. Available online: http://iesrj.com/journal/index.php/iesrj/ article/view/59 (accessed on 16 April 2016). 
12. Swan, M. The quantified self: Fundamental disruption in big data science and biological discovery. Big Data 2013, 1, 85-99. [CrossRef]

13. Oh, J.; Lee, U. Exploring ux issues in quantified self technologies. In Proceedings of the 2015 Eighth International Conference on Mobile Computing and Ubiquitous Networking (ICMU), Hakodate, Japan, 20-22 January 2015; pp. 53-59.

14. Patel, S.; Park, H.; Bonato, P.; Chan, L.; Rodgers, M. A review of wearable sensors and systems with application in rehabilitation. J. Neuroeng. Rehabil. 2012, 9, 1-17. [CrossRef] [PubMed]

15. Mostarac, P.; Malarić, R.; Jurčević, M.; Hegedu, H.; Lay-Ekuakille, A.; Vergallo, P. System for monitoring and fall detection of patients using mobile 3-axis accelerometers sensors. In Proceedings of the 2011 IEEE International Workshop on Medical Measurements and Applications Proceedings (MeMeA), Bari, Italy, 30-31 May 2011; pp. 456-459.

16. Wang, J.; Zhang, Z.; Zheng, Y.; Wu, M.; Kim, J.-U.; Prasad, K.; Sharma, R.; Wadhwani, D.; Ma, L.; Li, X. A novel fall activity recognition method for wireless sensor networks. Int. J. U- e-Service Sci. Technol. 2012, 5, $1-14$.

17. Lee, S.; Polito, S.; Agell, C.; Mitra, S.; Yazicioglu, R.F.; Riistama, J.; Habetha, J.; Penders, J. A Low-power and Compact-sized Wearable Bio-impedance Monitor with Wireless Connectivity. In Proceedings of the Journal of Physics: Conference Series, Heilbad Heiligenstadt, Germany, 22-25 April 2013; p. 012013.

18. Jensen, U.; Leutheuser, H.; Hofmann, S.; Schuepferling, B.; Suttner, G.; Seiler, K.; Kornhuber, J.; Eskofier, B.M. A wearable real-time activity tracker. Biomed. Eng. Lett. 2015, 5, 147-157. [CrossRef]

19. Chan, M.; Estève, D.; Fourniols, J.-Y.; Escriba, C.; Campo, E. Smart wearable systems: Current status and future challenges. Artif. Intell. Med. 2012, 56, 137-156. [CrossRef] [PubMed]

20. Mukhopadhyay, S.C. Wearable sensors for human activity monitoring: A review. IEEE Sens. J. 2015, 15, 1321-1330. [CrossRef]

21. Whitcup, M.S.; Lamattina, K. The Wearable Medical Device in Your Future Is Now! Available online: http: //www.marketingresearch.org/article/wearable-medical-device-your-future\%E2\%80\%A6is-now (accessed on 20 April 2016).

22. Vandrico Solutions Inc. Available online: http://vandrico.com/wearables/ (accessed on 13 January 2016).

23. Belknap, R.; Weis, S.; Brookens, A.; Au-Yeung, K.Y.; Moon, G.; DiCarlo, L.; Reves, R. Feasibility of an ingestible sensor-based system for monitoring adherence to tuberculosis therapy. PLoS ONE 2013, 8, e53373. [CrossRef] [PubMed]

24. DiCarlo, L.; Moon, G.; Intondi, A.; Duck, R.; Frank, J.; Hafazi, H.; Behzadi, Y.; Robertson, T.; Costello, B.; Savage, G. A digital health solution for using and managing medications: Wirelessly observed therapy. IEEE Pulse 2012, 3, 23-26. [CrossRef] [PubMed]

25. Hubanova, R.; Aptel, F.; Chiquet, C.; Mottet, B.; Romanet, J.P. Effect of overnight wear of the triggerfish ${ }^{\circledR}$ sensor on corneal thickness measured by visante ${ }^{\circledR}$ anterior segment optical coherence tomography. Acta Ophthalmol. 2014, 92, e119-e123. [CrossRef] [PubMed]

26. Senior, M. Novartis signs up for google smart lens. Nat. Biotechnol. 2014, 32, 856-856. [CrossRef] [PubMed]

27. Kim, D.-H.; Lu, N.; Ma, R.; Kim, Y.-S.; Kim, R.-H.; Wang, S.; Wu, J.; Won, S.M.; Tao, H.; Islam, A. Epidermal electronics. Science 2011, 333, 838-843. [CrossRef] [PubMed]

28. Silva, F.; Analide, C.; Novais, P. Traffic expression through ubiquitous and pervasive sensorization-smart cities and assessment of driving behaviour. In Proceedings of the 5th International Conference on Pervasive and Embedded Computing and Communication Systems, Angers, France, 11-23 Febrary 2015; pp. $33-42$.

29. Hussain, S.; Kang, B.H.; Lee, S. A wearable device-based personalized big data analysis model. In Ubiquitous Computing and Ambient Intelligence. Personalisation and User Adapted Services; Springer: Cham, ZG, Switzerland, 2014; pp. 236-242.

30. Evans, D. Cisco. Available online: http://blogs.cisco.com/ioe/wearable-to-aware-able-contact-connectioncontext (accessed on 13 January 2016).

31. Perera, C.; Zaslavsky, A.; Christen, P.; Georgakopoulos, D. Context aware computing for the internet of things: A survey. IEEE Commun. Surv. Tutor. 2014, 16, 414-454. [CrossRef]

32. Jeong, J.-S.; Bang, D.W. An unified representation of context knowledge base for mobile context-aware system. J. Inf. Process. Syst. 2014, 10, 581-588. [CrossRef]

33. Augusto, J.C.; Callaghan, V.; Cook, D.; Kameas, A.; Satoh, I. Intelligent environments: A manifesto. Hum.-Centric Comput. Inf. Sci. 2013, 3, 1-18. [CrossRef] 
34. Wang, D.; Lo, D.; Bhimani, J.; Sugiura, K. Anycontrol-iot based home appliances monitoring and controlling. In Proceedings of the 2015 IEEE 39th Annual Computer Software and Applications Conference (COMPSAC), Taichung, Taiwan, 1-5 July 2015; pp. 487-492.

35. Manzoor, A.; Patsakis, C.; Morris, A.; McCarthy, J.; Mullarkey, G.; Pham, H.; Clarke, S.; Cahill, V.; Bouroche, M. Citywatch: Exploiting sensor data to manage cities better. Trans. Emerg. Telecommun. Technol. 2014, 25, 64-80. [CrossRef]

36. Gubbi, J.; Buyya, R.; Marusic, S.; Palaniswami, M. Internet of things (iot): A vision, architectural elements, and future directions. Future Gener. Comput. Syst. 2013, 29, 1645-1660. [CrossRef]

37. Raychoudhury, V.; Cao, J.; Kumar, M.; Zhang, D. Middleware for pervasive computing: A survey. Pervasive Mob. Comput. 2013, 9, 177-200. [CrossRef]

38. Römer, K.; Kasten, O.; Mattern, F. Middleware challenges for wireless sensor networks. ACM SIGMOBILE Mob. Comput. Commun. Rev. 2002, 6, 59-61. [CrossRef]

39. Muro, M.; Amoretti, M.; Zanichelli, F.; Conte, G. Towards a flexible middleware for context-aware pervasive and wearable systems. Med. Biol. Eng. Comput. 2012, 50, 1127-1136. [CrossRef] [PubMed]

40. Ben Arbia, D.; Alam, M.M.; Attia, R.; Ben Hamida, E. Behavior of wireless body-to-body networks routing strategies for public protection and disaster relief. In Proceedings of the 2015 IEEE 11th International Conference on Wireless and Mobile Computing, Networking and Communications (WiMob), Abu Dhabi, United Arab Emirates, 19-21 October 2015; pp. 117-124.

41. Alam, M.M.; Hamida, E.B. Surveying wearable human assistive technology for life and safety critical applications: Standards, challenges and opportunities. Sensors 2014, 14, 9153-9209. [CrossRef] [PubMed]

42. Solanas, A.; Patsakis, C.; Conti, M.; Vlachos, I.; Ramos, V.; Falcone, F.; Postolache, O.; Perez-martinez, P.; Pietro, R.; Perrea, D. Smart health: A context-aware health paradigm within smart cities. IEEE Commun. Mag. 2014, 52, 74-81. [CrossRef]

43. Páez, D.G.; de Buenaga Rodríguez, M.; Sánz, E.P.; Villalba, M.T.; Gil, R.M. Big data processing using wearable devices for wellbeing and healthy activities promotion. In Ambient Assisted Living. Ict-based Solutions in Real Life Situations; Springer: Cham, ZG, Switzerland, 2015; pp. 196-205.

44. McLeroy, K.R.; Bibeau, D.; Steckler, A.; Glanz, K. An ecological perspective on health promotion programs. Health Educ. Q. 1988, 15, 351-377. [CrossRef] [PubMed]

45. Kazak, A.E. Families of chronically ill children: A systems and social-ecological model of adaptation and challenge. J. Consult. Clin. Psychol. 1989, 57, 25. [CrossRef] [PubMed]

46. Bergmann, J.H.; Chandaria, V.; McGregor, A. Wearable and implantable sensors: The patient's perspective. Sensors 2012, 12, 16695-16709. [CrossRef] [PubMed]

47. Itea3. Available online: https://itea3.org/innovation-report/improving-the-quality-of-everyday-lifethrough-wearable-computers.html (accessed on 13 January 2016).

48. Palmer, B.; Gershbein, D. The wearables for good challenge. Available online: http://wearablesforgood. com/WearablesForGood-UseCaseHandbook.pdf (accessed on 13 January 2016).

49. Whitehouse, B.; Panton, S. The wearables for good challenge. Available online: http://wearablesforgood. com/crossover-of-consumer-products-wearable-technology-for-social-good/ (accessed on 13 January 2016).

50. Bleser, G.; Steffen, D.; Reiss, A.; Weber, M.; Hendeby, G.; Fradet, L. Personalized physical activity monitoring using wearable sensors. In Smart Health; Springer: Cham, ZG, Switzerland, 2015; pp. 99-124.

51. Gilmore, J.N. Everywear: The quantified self and wearable fitness technologies. New Media Soc. 2015. [CrossRef]

52. Jovanov, E.; Milenkovic, A. Body area networks for ubiquitous healthcare applications: Opportunities and challenges. J. Med. Syst. 2011, 35, 1245-1254. [CrossRef] [PubMed]

53. Hadjidj, A.; Souil, M.; Bouabdallah, A.; Challal, Y.; Owen, H. Wireless sensor networks for rehabilitation applications: Challenges and opportunities. J. Network Comput. Appl. 2013, 36, 1-15. [CrossRef]

54. Qi, Y.; Soh, C.B.; Gunawan, E.; Low, K.-S. Ambulatory measurement of three-dimensional foot displacement during treadmill walking using wearable wireless ultrasonic sensor network. IEEE J. Biomed. Health Inform. 2015, 19, 446-452. [CrossRef] [PubMed]

55. Dobkin, B.H. Wearable motion sensors to continuously measure real-world physical activities. Curr. Opin. Neurol. 2013, 26, 602-608. [CrossRef] [PubMed] 
56. Lee, C.N.; Chen, L.L.; Chu, Y.T.; Lai, T.H.; Cheng, Y.C.; Cheng, L. A localization and safety monitoring cane. In Proceedings of the 2015 IEEE MTT-S 2015 International Microwave Workshop Series on RF and Wireless Technologies for Biomedical and Healthcare Applications (IMWS-BIO), Taipei, Taiwan, 21-23 September 2015; pp. 235-236.

57. Shany, T.; Redmond, S.J.; Narayanan, M.R.; Lovell, N.H. Sensors-based wearable systems for monitoring of human movement and falls. IEEE Sens. J. 2012, 12, 658-670. [CrossRef]

58. Ng, J.K.-Y. Ubiquitous healthcare: Healthcare systems and applications enabled by mobile and wireless technologies. J. Converg. 2012, 3, 31-36.

59. Manduchi, R.; Kurniawan, S. Mobility-related accidents experienced by people with visual impairment. AER J.: Res. Pract. Vis. Impair. Blind. 2011, 4, 44-54.

60. Madden, P.; Leaman, R.; Corrigan, N. The future cities catapult. Available online: http:/ /www.citiesunlocked. org.uk/wp-content/uploads/2014/11/CUReport_WEB.pdf (accessed on 13 January 2016).

61. Lee, J.H.; Kim, D.; Shin, B.S. A wearable guidance system with interactive user interface for persons with visual impairment. Multimed. Tools Appl. 2014, 1-22. [CrossRef]

62. Charara, S. Wearable. Available online: http://www.wareable.com/wearable-tech/wearable-tech-savesthe-day-from-exosuits-to-robotic-hands-to-nasa-glasses-772 (accessed on 13 January 2016).

63. Curone, D.; Dudnik, G.; Loriga, G.; Luprano, J.; Magenes, G.; Paradiso, R.; Tognetti, A.; Bonfiglio, A. Smart garments for safety improvement of emergency/disaster operators. In Proceedings of the 2007 29th Annual International Conference of the IEEE Engineering in Medicine and Biology Society, Lyon, France, 22-26 August 2007; pp. 3962-3965.

64. Fischer, C.; Gellersen, H. Location and navigation support for emergency responders: A survey. IEEE Pervasive Comput. 2010, 9, 38-47. [CrossRef]

65. Vallozzi, L.; Vandendriessche, W.; Rogier, H.; Hertleer, C.; Scarpello, M.L. Wearable textile gps antenna for integration in protective garments. In Proceedings of the 2010 Fourth European Conference on Antennas and Propagation (EuCAP), Barcelona, Spain, 12-16 April 2010; pp. 1-4.

66. Basich, M. Police Magazine. Available online: http://www.policemag.com/channel/technology/articles / 2015/04/robocop-wearable-tech.aspx (accessed on 13 January 2016).

67. Baker, P.; Gandy, M.; Zeagler, C. Innovation and wearable computing: A proposed collaborative policy design framework. IEEE Int. Comput. 2015, 19, 18-25. [CrossRef]

68. Kirkham, R.; Greenhalgh, C. Social access vs. Privacy in wearable computing: A case study of autism. IEEE Pervasive Comput. 2015, 14, 26-33. [CrossRef]

69. Paul, G.; Irvine, J. Privacy implications of wearable health devices. In Proceedings of the 7th International Conference on Security of Information and Networks, Glosgow, UK, 9-11 September 2014; p. 117.

70. Starner, T. How wearables worked their way into the mainstream. IEEE Pervasive Comput. 2014, 13, 10-15. [CrossRef]

71. Sung, D. Wearable. Available online: http://www.wareable.com/health-and-wellbeing/wearable-tech-andregulation-5678 (accessed on 13 January 2016).

(C) 2016 by the authors; licensee MDPI, Basel, Switzerland. This article is an open access article distributed under the terms and conditions of the Creative Commons Attribution (CC-BY) license (http://creativecommons.org/licenses/by/4.0/). 